\title{
Antenna Models for Electromagnetic Compatibility Applications
}

\author{
Dragan Poljak, ${ }^{1}$ Khalil El Khamlichi Drissi, ${ }^{2}$ \\ Sergey V. Tkachenko, ${ }^{3}$ and Andres Peratta ${ }^{4}$ \\ ${ }^{1}$ Department of Electronics, University of Split, Rudera Boskovica 32, 21000 Split, Croatia \\ ${ }^{2}$ Institut Pascal, UMR6602, Blaise Pascal University, Clermont Ferrand, France \\ ${ }^{3}$ Otto-von-Guericke University Magdeburg, Universitaetsplatz 2, 39106 Magdeburg, Germany \\ ${ }^{4}$ Wessex Institute of Technology, Ashurst Lodge, Ashurst, Southampton SO40 7AA, UK \\ Correspondence should be addressed to Dragan Poljak,dpoljak@fesb.hr
}

Received 13 February 2012; Accepted 13 February 2012

Copyright (C) 2012 Dragan Poljak et al. This is an open access article distributed under the Creative Commons Attribution License, which permits unrestricted use, distribution, and reproduction in any medium, provided the original work is properly cited.

In recent decades there have been a number of significant advances in EMC modeling which can be carried out within a significantly shorter time than it would be necessary for building and testing the appropriate prototype via experimental procedures. Moreover EMC simulation can predict the system behaviour for a rather wide variety of parameters including different initial and boundary conditions, excitation types, and different configuration of the system itself. EMC-computational models are often classified as

(i) circuit theory models (featuring the concentrated electrical parameters),

(ii) transmission line models (using distributed parameters in which low-frequency electromagnetic field coupling is taken into account), antenna systems, overhead power lines, and buried cables.

(iii) models based on the full-wave (antenna theory) approach (taking into account radiation effects for the treatment of electromagnetic wave propagation problems).

The antenna models represent a vital area of EMC related to the study of electromagnetic field coupling to systems or devices and represent the most rigorous approach to EMC problems. Furthermore, the transmission line (TL) approximation, whose principal advantage is simplicity and relatively low computational cost, fails to account for various radiation effects, such as resonances, presence of a lossy ground, the effects at the wire ends, and so forth However, the main restriction of the wire antenna model applied to complex structures is related to a rather high computational cost.

Therefore, the Guests Editors enthusiastically accepted the opportunity to edit this special issue on antenna models for Electromagnetic Compatibility Applications by collecting some interesting contributions achieved by some researchers in this area. This special issue deals with various aspects of antenna EMC models covering applications related to both continuous wave $(\mathrm{CW})$ problems and transient problems.

Guest Editors have received 11 submissions for this Special issue. Having completed the peer review process, the Guest Editors have finally accepted 5 papers, while 5 papers have been rejected and 1 paper has been withdrawn.

The issue entitled "Electromagnetic Field Coupling to Overhead Wire Configurations", by D. Poljak and K. ElKhamlichi Drissi reviews various aspects of wire antenna and transmission line models in both frequency and time domain. The paper by S. Tkachenko et al. entitled "High Frequency Electromagnetic Field Coupling to Small Antenna in a Rectangular Resonator" deals with an efficient analytical approach to analyze the coupling of electrically small antennas in resonators. The paper "Modeling of Coaxial Slot Waveguides Using Analytical and Numerical ApproachesRevisited" written by Y. K. You et al. reviews some analytical and numerical models for coaxial slot waveguides. Paper: "Evaluation of the Inductive Coupling between Equivalent Emission Sources of Components" by M. Ferber et al. presents methodology providing the evaluation of coupling 
parameters of components by using equivalent emission sources. Finally, the paper written by V. Javor and entitled "Modeling of Lightning Strokes using Two-Peaked ChannelBase Currents." is related to a study of lightning induced electromagnetic field by means of engineering models of lightning return strokes and new channel-base currents.

The Guest Editors hope they managed to put together an interesting piece of work regarding the use of antenna models in electromagnetic compatibility applications.

They would also like to thank all contributors for their valuable contributions and to the reviewers for their prompt response and excellent work, as well.

Dragan Poljak

Khalil El Khamlichi Drissi

Sergey V. Tkachenko Andres Peratta 

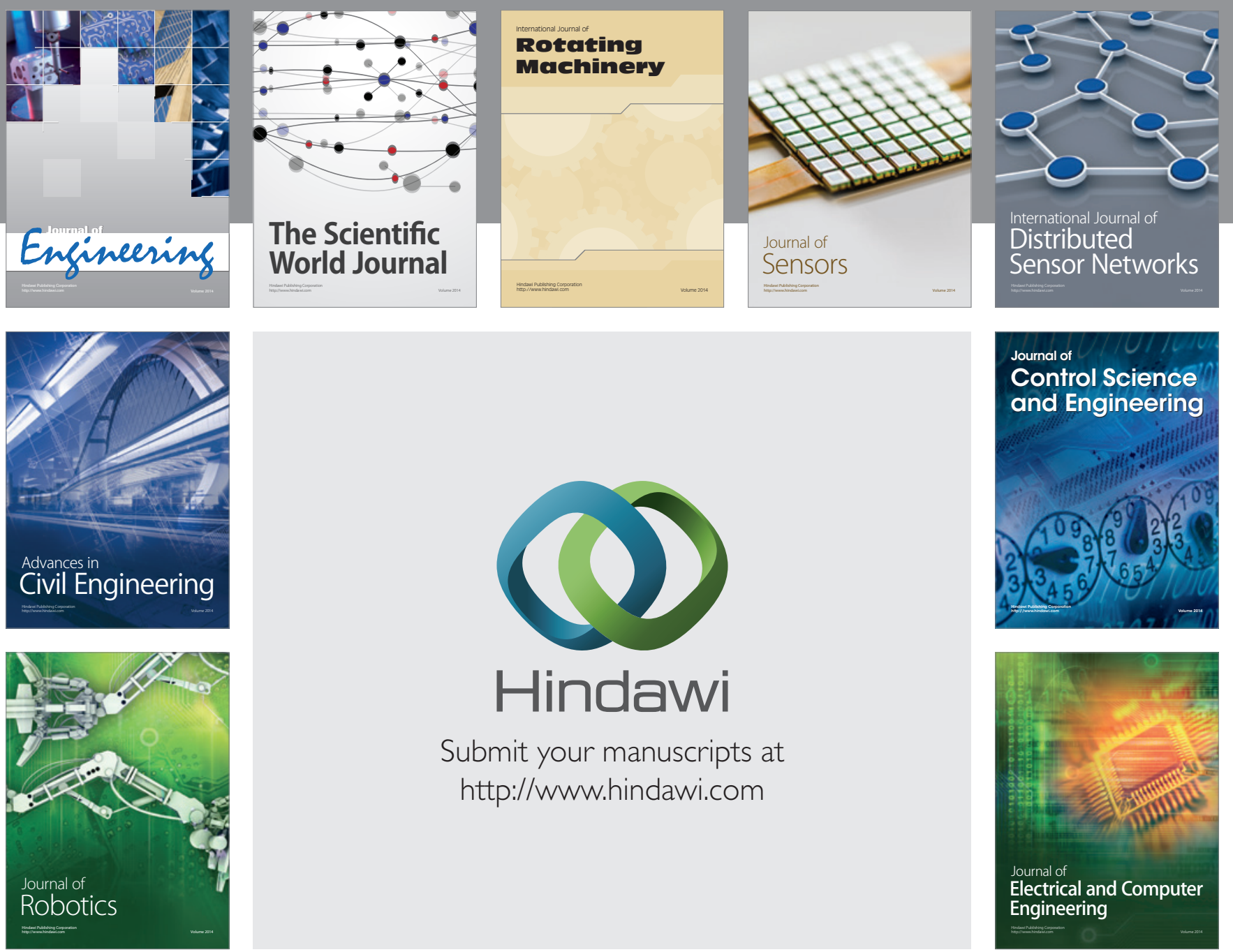

Submit your manuscripts at

http://www.hindawi.com
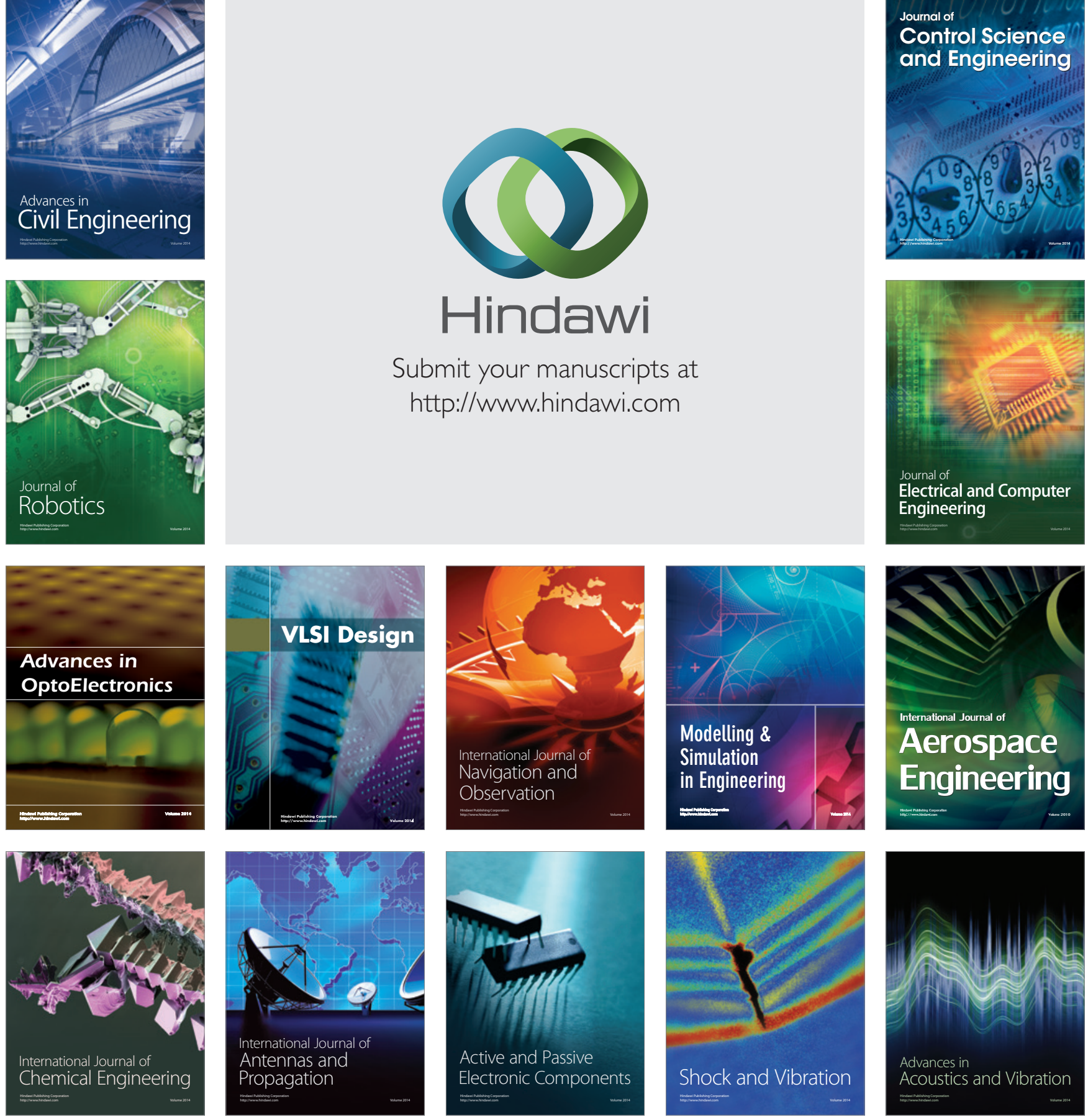